\title{
VIVENCIA DE ESTUDIANTES DE ENFERMERÍA DE LA TRANSICIÓN A LA PRÁCTICA PROFESIONAL: UN ENFOQUE FENOMENOLÓGICO SOCIAL
}

\author{
Antonia Vollrath Ramírez', Margareth Angelo², Luz Angélica Muñoz González
}

\footnotetext{
${ }^{1}$ Magíster en Enfermería. Profesor Asistente de la Escuela de Enfermería de la Facultad de Enfermería de la Universidad Andrés Bello. Santiago de Chile, Chile. E-mail: avollrath@unab.cl

${ }^{2}$ PhD. Profesora Titular da Escola de Enfermagem da Universidade de São Paulo. São Paulo, Brasil, E-mail: angelm@usp.br

${ }^{3}$ PhD. Profesora Titular de la Facultad de Enfermería de la Universidad Andrés Bello. Santiago de Chile, Chile, E-mail: 1munoz@ unab.cl
}

RESUMEN: La finalidad de este estudio fue comprender el significado de la experiencia de aprendizaje de transición del estudiante de enfermería, y el significado de sus expectativas del aprendizaje para su futura práctica profesional. Optamos por una investigación cualitativa, con enfoque fenomenológico social de Alfred Shutz, para lo cual se entrevistó a diez internos de enfermería. Del análisis comprensivo del tipo vivido, fue identificado que el estudiante siente que al asumir responsabilidades experimenta sufrimiento e inseguridad por falta de conocimiento, no confía en sus habilidades y se siente solo; pero a medida que percibe apoyo adquiere confianza, autonomía y se integra al equipo. Siente además que la enfermera es importante para la transición. Se lograron comprender en el proceso de transición los motivos para y los motivos por qué de sus acciones, y cómo se integra al mundo de la vida cotidiana de un determinado grupo social en un determinado tiempo y espacio.

DESCRIPTORES: Enfermería. Educación. Aprendizaje.

\section{THE EXPERIENCE OF NURSING STUDENTS IN THEIR TRANSITION TO PROFESSIONAL PRACTICE: A SOCIAL PHENOMENOLOGICAL APPROACH}

\begin{abstract}
The purpose of this study was to better understand the meaning of the learning experience in transitioning from nursing student to professional, and the meaning of their expectations from their learning experience upon their future professional practice. The social phenomenological approach of Alfred Schutz was adopted in interviewing ten nursing interns. Comprehensive living experience analysis identified that the student feels that they take on suffering and uncertainty in taking on responsibility due to their lack of knowledge, lack of confidence in their abilities, and feelings of solitude. However, to the degree they perceive support they gain confidence, autonomy, and integrate themselves with the team. He/she feels, however, that nurses are important for the transition. This study made it possible to understand the reasons and motives for their actions within the transition process, as well as how a particular social group in a given time and space is integrated into the world of everyday life.
\end{abstract}

DESCRIPTORS: Nursing. Education. Learning.

\section{EXPERIÊNCIA DOS ESTUDANTES DE ENFERMAGEM NA TRANSIÇÃO PARA A PRÁTICA PROFISSIONAL: UMA ABORDAGEM SOCIAL FENOMENOLÓGICA}

RESUMO: Os objetivos deste estudo foram compreender o significado da experiência de aprendizagem de transição do estudante de enfermagem e o significado de suas expectativas de aprendizagem para a futura prática profissional. A abordagem da Fenomenologia Social de Alfred Schutz foi utilizada na condução da pesquisa. Foram entrevistados dez estudantes de internato de enfermagem. A análise compreensiva do tipo vivido permitiu identificar que o estudante sente que, ao assumir responsabilidades, experimenta sofrimento e insegurança devido à falta de conhecimento, não confia em suas habilidades e se sente sozinho; mas à medida que identifica apoio, ganha confiança, autonomia, integra-se à equipe e sente que o enfermeiro é importante para a sua transição. O estudo permitiu compreender no processo de transição, os motivos para e os motivos porque das ações do estudante, e como ele se integra no mundo da vida cotidiana de um determinado grupo em um determinado tempo e espaço.

DESCRITORES: Enfermagem. Educação. Aprendizagem. 


\section{INTRODUCCIÓN}

La trayectoria de este estudio comienza desde el siguiente fenómeno: El aprendizaje de transición del estudiante de enfermería cursa la asignatura de gestión en salud familiar y comunitaria (internado). Frente a este fenómeno surgen muchas inquietudes: ¿Cómo el estudiante percibe la transición en la asignatura de gestión en salud familiar y comunitaria (internado)?; ¿Cuáles son los sentimientos vividos?; ¿Qué siente al ser considerado como estudiante en ciertas ocasiones y como enfermero en otras?; ¿Es importante el apoyo de enfermera guía para su aprendizaje?; ¿Cómo enfrenta el cuidado de las personas incluyendo a la familia, la comunidad, las redes, desde una posición con y sin responsabilidad profesional?

Si tomamos en cuenta que la estructura de la enseñanza de enfermería persigue obtener profesionales altamente capacitados que respondan a las necesidades de salud en la sociedad, el programa de educación de enfermería está basado en las necesidades de salud y la prevención de la enfermedad así como el cuidado y autocuidado ante la pérdida de la salud en las diferentes etapas de vida. Por consiguiente, se espera que los estudiantes de enfermería dominen ciertas habilidades esenciales del hacer profesional, como lavado de manos, baño de esponja; toma, registro y valoración de signos vitales (temperatura, pulso, respiración y presión arterial) así como numerosos procedimientos de asepsia y antisepsia, entre otros procedimientos. ${ }^{1}$

Las transiciones son complejas y multidimensionales. Son un resultado de los cambios en la vida, salud, relaciones y entornos. ${ }^{2}$ Creemos que es importante conocer las experiencias vividas por los estudiantes de enfermería que se están preparando para cuidar de personas, a fin de adecuar el currículum a las metodologías y estrategias de enseñanza, que permitan la formación de profesionales competentes. ${ }^{3}$

La enfermera y los profesores por mucho tiempo han reconocido que la práctica clínica es muy significativa, y es la parte esencial de la educación de los estudiantes de enfermería. La complejidad de lo que los estudiantes de enfermería deben aprender puede que sólo sea desarrollado con la exposición continua directa para el trasfondo clínico. ${ }^{4}$

Se logró percibir que los estudiantes durante la formación se impregnan de comportamientos, actitudes y valores, lo que determina la construcción del rol. A través del tiempo acumulan diversas situaciones vividas, ya sea en la relación con otros estudiantes, pacientes, enfermera docente y enfermera clínica, considerando que la experiencia de esta asignatura de internado ocasiona cambios en el comportamiento y en el desarrollo de una actitud social profesional como fin de integrar las cuatro funciones del rol.

En virtud de lo anterior, los objetivos del estudio fueron: conocer y comprender el significado de la experiencia de aprendizaje de transición del estudiante de enfermería (internado), y comprender el significado de sus expectativas del aprendizaje de esa experiencia para su futura práctica profesional.

\section{METODOLOGÍA}

La investigación cualitativa posibilita que el investigador capte la reacción de los individuos en su propia realidad. Trabaja con el universo de significados, valores, y actitudes de los individuos, permitiendo al investigador conocer la dinámica y la situación desde el punto de vista de quienes la vivencian. ${ }^{5}$

En la investigación fenomenológica, el investigador está preocupado con la naturaleza de lo que va investigar, por lo que no existe una comprensión previa del fenómeno. ${ }^{6}$ El referente de este estudio el enfoque de la fenomenología social Alfred Schütz; ${ }^{7-10}$ a través de su teoría de comprensión de la acción social, se permitió comprender al estudiante, desde sus motivos para y los motivos por qué de sus acciones, así como comprender cómo se integra al mundo de la vida cotidiana de cierto grupo social en un determinado tiempo y espacio. La situación biográfica de cada estudiante aporta su equipaje de conocimientos, sus experiencias y la vivencia de una relación cara a cara con la enfermera guía, con el paciente y su familia, lo que otorga comprender una comprensión sobre cómo se desarrolla la acción social y la intersubjetividad con el equipo de profesionales. Esto permite investigar tanto los comportamientos individuales como grupos sociales que viven una situación típica, ya que permite develar el sentido del mundo cotidiano de ser y estar con el otro, contextualizando una intersubjetividad y configurando un grupo social..$^{7-10}$

El mundo de la vida cotidiana también es desde el principio un mundo social y cultural dentro del cual el sujeto se relaciona, de múltiples formas de interacción con los semejantes que se conocen en diversos grados de intimidad y anonimato. Este conocimiento es para fines prác- 
ticos, donde la comprensión de sus conductas, sus motivos, objetivos y planes que se originan en circunstancias biográficamente determinadas.

Para el desarrollo de la investigación, fue utilizada como región de indagación la asignatura de Gestión en Salud Familiar y Comunitaria de la Facultad de Enfermería de la Universidad Andrés Bello, que es el lugar donde se vivencia la experiencia, y desde donde emergen las inquietudes que se buscan develar con este estudio. El objetivo de esta asignatura es que el estudiante logre ejercer el rol profesional bajo supervisión y guía docente asistencial en comunidad; además, debe integrarse al trabajo con el equipo de salud en los centros asistenciales asignados por la Escuela. Es aquî donde las y los estudiantes vivencian experiencias de enseñanza - aprendizaje de transición con el gran desafío de asumir el rol profesional.

A través de una comunicación vía correo electrónico, se invitó a participar voluntariamente en este estudio a todos los estudiantes que habían finalizado la asignatura. La propuesta fue visada por el comité de ética científico de la Universidad, el cual autorizó el estudio. Previo a realizar las entrevistas se procuró crear un ambiente favorable, a fin de que cada entrevistado se sintiera libre para contar su experiencia. Antes de cada entrevista fue presentado el objetivo del estudio y posteriormente se entregó el consentimiento, el cual tras ser leído, y en caso de estar de acuerdo, requería ser firmado.

Para la recolección de datos se utilizaron entrevistas individuales, creando condiciones para que el sujeto se expresara libre y espontáneamente, relatando sus experiencias en forma precisa y descriptiva, tal como la perciben ellos. En el estudio fueron utilizadas dos preguntas orientadoras: ¿Cómo fue para usted el aprendizaje de transición en la asignatura de Gestión en Salud Familiar y Comunitaria? y Ahora al término de esta asignatura. ¿Qué espera usted de esta experiencia aprendida, para su práctica profesional?

Los sujetos de estudio fueron diez internos de enfermería, seis estudiantes del año 2007, y cuatro estudiantes del año 2008, siendo nueve de sexo femenino y uno de sexo masculino. Las edades fluctúan entre 24 y 31 años y aceptaron participar voluntariamente, firmando un consentimiento informado con los resguardos éticos pertinentes.

En la etapa de análisis de datos, se realizó la lectura y relectura de la trascripción del material, tratando de escuchar atenta y cuidadosamente el discurso de los sujetos en sus expresiones natura- les. Se continuó con el análisis de los datos transcritos buscando convergencia de los "motivos para qué" (proyectos de futuro) y los "motivos por qué" (contextos de motivación), vividos en los temas que llevaron a cabo la acción del aprendizaje de transición de los estudiantes de la asignatura Gestión en Salud Familiar y Comunitario (internado).

El desarrollo de un sistema ordenado de los contenidos significativos implica varios pasos y exige una organización sistematizada que permitió responder el objetivo del estudio y las preguntas orientadoras. Para proceder al análisis individual de los discursos se utiliza el recorrido metodológico de la fenomenología social, que no encierra una descripción de lo subjetivo sino que convive con lo intersubjetivo, permitiendo la comprensión e interpretación que no es del investigador, sino que abarca lo histórico-social y contexto de lo investigado. $^{11}$

Al recorrer el camino para la construcción de categorías concretas de lo vivido, el objetivo del estudio, el referencial filosófico a Schütz ha permeando con esta filosofía destinada a comprender las motivaciones constituyentes de la percepción de los estudiantes de internado comunitario. Sobre esta base es posible captar el tipo expresado en los discursos y constituir el tipo vivido por el estudiante, en relación a la percepción que tiene del aprendizaje.

\section{RESULTADOS}

Para una mejor comprensión de esta experiencia, esta es descrita con cinco categorías que emergieron del análisis de los datos: Asumir la responsabilidad del rol profesional; Sentirse reconocido en su desempeño profesional; Tener a la enfermera como guía en la transición; Sensibilizarse con el mundo de los pacientes; y Viéndose como enfermera y como persona.

A continuación se presenta cómo de cada categoría emergen los "motivos por qué" y "motivos para" en la vivencia del estudiante:

\section{Asumir la responsabilidad del rol profesional (motivos por qué)}

El estudiante reconoce que el conocimiento influye en su desempeño y se traduce en emociones y sentimientos. Estos tienen un efecto tanto en la experiencia de la transición como en el resultado de esta. El sufrimiento es una emoción que se presenta de manera más intensa al inicio de la 
vivencia. Es un desafío para el estudiante quedarse solo, en este proceso de transición entre el rol de estudiante y el rol profesional. Esta emoción al inicio de la experiencia de transición es la que predomina en este periodo, resultante de la gran cantidad de labores y roles que este debe realizar:

[...] sí, entonces igual a mí me daba... como... un poco de temor eso porque, si yo me equivocaba, o demás que me habré equivocado en algunas cosas, viendo tantos pacientes al mismo tiempo, entonces eso me estresaba, equivocarme, si tuviera que definir en una palabra fue [...] un internado muy sola [ríe] yo sola, autodidacta, no sé cómo podría definirlo... como fui aprendiendo a porrazos sentí en parte pena e impotencia, sentí que [...] no podía hacer tantas cosas (D2).

La sensación de falta de conocimiento provoca un temor a cometer errores en su desempeño, sensación de impotencia frente a situaciones que cree que debe resolver, temor de no ser reconocida como profesional por el personal de enfermería y por los pacientes. También influye cuando tiene que enfrentar nuevas tareas y la gran cantidad de responsabilidades que debe asumir. El conocimiento juega un rol fundamental en la toma de decisiones del estudiante. Primero, aumenta el nivel de confianza y les permite sentir que el puede manejar la situación y tomar decisiones seguras e independientes:

[...] es como, que es difícil en el fondo en el sentido de que... uno es alumna a veces y en otras como ya enfermera, de hecho nosotros el primer día que llegamos las enfermeras tenian unas reuniones y nos dejaron a nosotras solas, el primer día en el mesón con una paciente nosotras hace [...] dos años que no veíamos consultorio, entonces igual la experiencia en el fondo igual nos faltaba y necesitábamos ver controles pronto, aunque si en el fondo no sean tan complejos yo necesitaba verlos y verte como enfrentada de repente a que te digan ya siéntate aqui ahora a ti te toca hacer los controles y [...] ahí con mi compañera nos dio miedo y de hecho nos llamábamos y ella estaba en su box que ni siquiera estábamos juntas y eso (D1).

\section{Sentirse reconocido en su desempeño profesional (motivos para)}

La satisfacción es una emoción que emerge en cuanto avanza la experiencia de internado y el estudiante siente confianza en enfrentar los desafíos del rol profesional. A medida que va desarrollando destrezas y habilidades organizacionales comienza a surgir un sentimiento de satisfacción. Los "motivos para" están determinados por los siguientes componentes: adquirir confianza en si mismo aumenta a medida que desarrolla destrezas organizacionales, marcando sus prioridades. El estudiante encuentra su propio estilo y ritmo, comienza a sentir una conexión con el equipo y con el centro de salud. Cuando el estudiante se siente aceptado, su percepción de la experiencia es significativamente diferente. El apoyo es percibido a través de las interacciones con las enfermeras tutoras cuando estas les amparan, dando aporte $\mathrm{y}$ fortaleciendo sus conocimientos y habilidades.

El reconocimiento es percibido a través de las interacciones con los pacientes, quienes demuestran satisfacción con los cuidados entregados por el estudiante, y también por las interacciones con el equipo de salud, cuando se siente aceptado o integrado como un miembro del equipo:

[...] súper gratificada, porque no sé qué será que dé confianza a las personas, porque ya el hecho de tener un uniforme de estudiante quita un poco de esa confianza, pero de repente es quizás la apertura para decir las cosas y la seriedad, no sé qué será, quizás la postura o el profesionalismo al tomar la conducta de enfermera, bueno la verdad es que nosotros en general sí había mucha confianza (D8).

La capacidad de integrar todo lo aprendido es percibida por el estudiante como una oportunidad de autonomía, obteniendo resultados en sus acciones junto a los pacientes. El estudiante tiene el objetivo de saber responder a tiempo a las necesidades de los pacientes. Otro componente es la integración al equipo de salud en el centro de salud donde el estudiante realiza su internado para asumir el rol profesional con confianza. La integración es una necesidad que posibilita al estudiante a sentirse parte del equipo porque el estar solo frente a un equipo ya formado constituye un desafío:

[...] yo me sentía una enfermera más [risas], me sentía súper confiada, tranquila, haciendo mi pega relajada, obviamente cumpliendo los horarios y todo eso, de repente súper apurada al principio sobre todo para tratar de hacer los controles a tiempo y programarme en las visitas y lograr salir y hacer todo de acuerdo a un cronograma establecido, pero después cuando uno se conoce y sabe cómo trabaja ya es distinto, o sea, súper fluida, no, pero me sentía como tranquila, como feliz en mi trabajo, muy contenta (D8).

La vivencia de lograr la integración al equipo de salud ocurre cuando el estudiante se percibe capaz de integrarse al equipo. Esta percepción se manifiesta cuando el estudiante identifica que no tiene problemas para trabajar con el equipo, cuando consigue participar en las reuniones de 
trabajo presentando sus ideas y construyendo buenas relaciones con el equipo de trabajo.

A míse me ha hecho súper pesado pero me gusta$b a, y$ yo siento que sí aprendí y me gustó y me tocaron, gracias a Dios, buena gente en el trabajo, enfermeros, un buen grupo, asistentes sociales, los técnicos, los médicos y que todos iban ayudando a ver si teníamos alguna duda, te ayudaban, las cosas que uno veía, no sé, me cuesta esto, siempre me ayudaban, había un apoyo, síhabía un apoyo y a mi me gustó bastante. Me gusta harto, encuentro que es mucha información, es mucho lo que hay que saber, pero para mí fue súper enriquecedor (D6).

\section{Tener a la enfermera como guía en la transición (motivos para)}

Tener a la enfermera como guía es un elemento facilitador para que el estudiante logre alcanzar la transición a ser enfermera. La relación de apoyo y confianza que el estudiante percibe en la enfermera actúa facilitando el desarrollo del rol profesional y la identidad de enfermera. El estudiante percibe que la enfermera guía ayuda a tornarse profesional de la salud cuando entrega las herramientas para realizar su quehacer, ofrece oportunidades para que adquiera la seguridad en la toma de decisiones que necesita para desenvolverse solo y facilita el camino demostrando confianza en su desempeño. Para alcanzar la transición de ser enfermera, el estudiante es facilitado cuando el percibe que la enfermera crea condiciones para su acogida por el equipo, lo que favorece su desarrollo como enfermera. La confianza se construye entre las enfermeras y el estudiante mediante el trabajo en conjunto y el aprendizaje mutuo en este período de tiempo, en el ambiente clínico lo cual favorece el proceso para alcanzar la transición de ser enfermera:

[...] lo más importante del internado es la relación que se mantiene con la enfermera guía del campo clínico, en mi caso fue muy significativo, fue una buena relación que mantuve con ambas enfermeras clínicas que estuvieron a mi cargo. Ellas yo creo que me dieron el apoyo...que es fundamental, para uno tener [...] la base y tener la seguridad, el poder sentirse buena, poder preguntarle cosas y en poder desenvolverse, porque al final ellas son [...] como [...] una madre [ríe] (D3).

\section{Sensibilizarse con el mundo de los pacientes (motivos para)}

Es la toma de conciencia de la responsabilidad frente a los cuidados que entrega a los pacien- tes como profesional. La relación profesional que el estudiante establece con el paciente y su familia en el internado estimula el conocimiento de sí mismo frente al mundo del paciente. El aprendizaje de compartir con ellos como profesionales crea una conexión especial de respeto a la percepción de vulnerabilidad de las personas que buscan ayuda para satisfacer sus necesidades físicas, sicológicas y sociales. Durante el encuentro con el paciente, el estudiante se enfrenta con muchas demandas, emociones y expectativas. Los pacientes representan una fuente de ayuda, conflicto, miedo, intimidación y conocimiento. Influyen en todo ámbito de la toma de decisiones del estudiante, incluyendo el cómo determinar la necesidad de una decisión clínica:

[...] una persona que vivía sola en una casa que estaba solamente entregada para el cuidado, esa persona no podía vivir fuera, no teníamos quién lo viera, entonces era una persona de riesgo, producto de todo lo que se puede vivenciar dentro de la casa; muchas situaciones me tocaron y a veces uno se pregunta, de qué forma uno puede tener la "varita" y tocar a esa gente que ha entregado mucho (D7).

\section{Viéndose como enfermera y como persona (motivos para)}

Es la visión del estudiante acerca de su vocación profesional, la consolidación de su identidad profesional y la proyección de lo que quiere para su futuro. El estudiante, comienza a tener una visión de proyección profesional y personal, analiza los modelos de enfermeras, las diferentes áreas de actuación profesional, y selecciona lo que quiere para su futuro. La excelencia y la capacidad de producir cambios son los elementos de gran motivación a la vida profesional futura de los estudiantes. Al verse a sí mismo como profesional, el estudiante reflexiona sobre el desarrollo de la seguridad en sí mismo y los desafíos para tornarse en el profesional que el desea. El estudiante se da cuenta cuán lejos ha llegado, empieza a confiar en su propio juicio y aumenta su percepción acerca de quién puede llegar a ser. A pesar del sufrimiento vivido al inicio de la orientación, el estudiante encuentra gran significancia y valor en su trabajo. El estudiante se proyecta a futuro asumiendo el rol profesional con capacidad de lograr excelencia y la capacidad de producir cambios en los pacientes a través del autocuidado, confiando en la autonomía, protagonismo y responsabilidad que los pacientes deben tener en el cuidado de su salud. 
Enfermería tiene mucho que entregar y las personas están muy desesperadas por salud y es verdad de que nosotros tenemos una deuda pendiente con estas personas. Espero que un futuro no lejano, porque los adultos mayores no pueden esperar un futuro lejano, porque ellos necesitan una atención ahora. Podemos solucionarle, ayudarle, hacer un poquito liviano, más gratificante su pasar y hacerles sentir de que ellos todavía son un ser que se valora, que tiene posibilidades en esta vida, que ya no están insertas dentro de un hoyo en el cual se tienen que tapar porque ya no sirve. Creo que enfermería tiene esa labor (D7).

Es importante considerar que las habilidades del estudiante aumentan a medida que avanza el periodo de internado, su percepción cambia, se sienten confiados en sus destrezas técnicas y de comunicación, experimentando satisfacción y asumiendo el rol profesional. El estudiante es capaz de construir sobre sus propias experiencias de aprendizaje para comenzar a entender el cuidado, reconociendo el patrón de respuestas de cada paciente y aprendiendo del paciente como una persona, tomando además conciencia que sus decisiones son las apropiadas. El hecho de que el estudiante aprenda a conectarse con el paciente significa lograr conocer al paciente al mismo tiempo en que el paciente le enseña al estudiante el "cómo" de la enfermería para comprometerse con la realidad de los pacientes. El paciente entrega al estudiante una gran cantidad de señales clínicas verbales y no verbales en relación del estudiante con el paciente.

El tipo vivido emerge de la descripción desde el comportamiento social, permitiendo encontrar algo que tipifique a las convergencias a las intenciones de los sujetos, o sea, "motivos para" y "motivos por qué". Esto, como una estructura vivenciada de forma única, uniforme, continua, que puede ser analizada y descrita ya que posee un valor de significado y puede ser transmitida por la comunicación y por el lenguaje significativo, la relación interpersonal reducida a los motivos típicos. En el tipo vivido del estudiante de enfermería que vivencia la asignatura de internado comunitario, el proceso de aprendizaje se construye cuando el estudiante siente que al asumir responsabilidades experimenta sufrimiento e inseguridad por la falta de conocimiento, no confía en sus habilidades y se siente solo; pero a medida que percibe ser apoyado por el equipo de salud, adquiere confianza, tiene más autonomía y se integra al equipo. Siente que la enfermera es una guía para la transición de ser estudiante a ser enfermera, así como al sensibilizarse con el mundo cotidiano de los pacientes se percibe como enfermera y como persona.

\section{DISCUSIÓN}

$\mathrm{Al}$ integrarse al mundo de la vida cotidiana del centro de salud, el estudiante tiene conciencia de que su experiencia es limitada y sabe que en esta etapa precisa ser capaz de integrar todos los conocimientos construidos a lo largo de los años de formación. Está conciente de que aun hay falta de conocimiento, lo que le genera emociones de sufrimiento, más intensas a un inicio, pero a medida que adquiere confianza en sí mismo comienza a asumir el rol profesional, lo que otorga emociones de satisfacción. Las personas actúan en función de la motivaciones dirigidas a objetivos que apuntan para el futuro, denominados "motivos para", siendo que las razones para sus acciones están enraizadas en experiencias del pasado, en la personalidad desarrollada durante su vida, llamadas "motivos por qué". 12

Al final de la formación profesional el proceso de transición de estudiante a enfermera es un rito común experimentado por todas las enfermeras egresadas. Es esencial que el estudiante sea aceptado con sus limitaciones y conflictos, siendo acompañado en el sentido de promover su madurez personal y profesional. La formación no es estrictamente técnica en su profesión, sino contemplando además la formación de los ciudadanos, persona - humano, cuyo hacer siempre tiene implicancias en la dimensión en la social y existencial. ${ }^{13}$

El punto final de una transición se alcanza cuando el individuo llega a un periodo en donde la sensación perturbadora de la transición ha disminuido y emerge un sentido de orden y estabilidad. Experiencias tales como la recuperación de la comodidad y la confianza, así como una señal de la reducción de desorden y un retorno de la estabilidad, tanto el papel psicológico y relacionado con el trabajo, sugiere una transición saludable. ${ }^{14}$

Los fenómenos de ajustes que se suscitan en el proceso de transición de estudiantes a enfermeras, deben desarrollar la capacidad de "pasar a través de", porque precisan socializar en la práctica de enfermería clínica y sus nuevas funciones profesionales. Los ajustes sobre este fenómeno han sido descritos tanto en la presentación de la tensión como en el desafío. Se describe el concepto de "choque con la realidad" como nuevo graduado al entrar en funciones de enfermería clínica, para encontrarse a sí mismo suficientemente preparados en las funciones que creía estar suficientemente preparado. Ansiedad, sentimientos de estar excesivamente abrumado y aprehensión son los sentimientos que más comúnmente surgen. ${ }^{15}$ 
No obstante, los autores coinciden en que el mentor es un modelo, maestro, entrenador, patrocinador, guía, consejero y, en general es mayor, más sabio, con más experiencia y más potente. ${ }^{16-17}$ Una desconexión entre las expectativas y la realidad de la experiencia fue un hallazgo común en la literatura revisada. ${ }^{18}$

La preparación para la transición de la práctica profesional de enfermería comienza cuando los estudiantes se acercan al final de su clínica supervisada y dan cuenta de que pronto se es responsable y deben rendir cuentas de su propia práctica como una enfermera registrada. La transición de ser una estudiante de enfermería para convertirse en un profesional de enfermería es la vez desafiante y estresante. ${ }^{19}$ Los mentores o educadores también podrían proporcionar información a través del foro en línea. Tópicos relacionados con los temas podrían ser presentados en todo el proceso de orientación como discusiones de grupo o por medio de un boletín informativo para los nuevos graduados. Atender las necesidades de los nuevos graduados de forma proactiva puede ser una poderosa herramienta para ayudar a crecer y las nuevas enfermeras prosperar en el mundo de la enfermería. ${ }^{19}$

Finalmente, en la investigación cualitativa realizada, el abordaje fenomenológico proporcionó respuestas sobre el significado de la experiencia de aprendizaje del estudiante de enfermería en su etapa de transición, y mostró los significados de sus expectativas de aprendizaje para su futura práctica profesional. Se logró comprender las acciones humanas, la experiencia del día a día de gente común, la vida cotidiana, las interrelaciones entre las personas y el hecho de conocer y ser conocido. Las implicaciones para enfermería fueron: sensibilizar a los profesores guías sobre una docencia facilitadora, contar con un programa de acompañamiento para este proceso de transición, considerar instancias para que el estudiante describa cómo está vivenciando su experiencia (foros). Dar a conocer estudios en profundidad a enfermeras clínicas para que adquieran conocimiento del complejo proceso de transición del estudiante, evaluar los entornos clínicos existentes, para que cumplan con las condiciones necesarias para el desarrollo del rol de enfermera y, por último, evaluar la malla curricular con el fin de entregar las herramientas requeridas durante su formación, para que este proceso sea significativo y positivo.

La interacción entre estudiantes y enfermeras devela una parte del fenómeno, que a cada mirada vuelve a develarse y a presentarse de manera distinta. Es importante continuar la búsqueda de la comprensión de los fenómenos que se presentan al formación de los estudiantes de enfermería desde la fenomenología, porque contribuye el conocimiento que involucra la experiencia del otro, y permite a ambos ser y ofrecer un cuidado auténtico a sus necesidades y expectativas. ${ }^{20}$

\section{REFERENCIAS}

1. Hernández E, Ramírez A. Los modelos interactivos para la enseñanza de habilidades en enfermería. Avances de proyecto financiado por el Programa de Apoyo a Proyectos institucionales para el mejoramiento de la enseñanza. clave EN208204 en la FES-Iztacala, UNAM. [citada 2009 May 26]. Disponible en: http:/ / www.salvador.edu.ar/vrid/ publicaciones/revista/1-tres.pdf

2. Meleis AI. Theoretical nursing. Development and progress. $3^{\mathrm{a}}$ ed. Philadelphia (US): Lippincott Williams\& Wilkins; 2005. p.93-101.

3. Pettengill MA, Angelo M. O sentido do cuidar da criança e da família na comunidade: a experiência da aluna de enfermagem. Rev Esc Enferm USP [online]. 2000 Mar [cited 2009 Jun 06]; 34(1):91-8. Disponible en: http:/ / www.scielo.br/pdf/reeusp/ v34n1/v34n1a12.pdf

4. Moya JLM, Parra SC. La enseñanza de la enfermería como una práctica reflexiva. Texto Contexto Enferm [online]. 2006 Abr-Jun [cited 2009 June 04]; 15(2):30311. Disponible en: http://redalyc.uaemex.mx/ redalyc/pdf/714/71415215.pdf

5. Merighi MAB, Praça NS. Abordagens teóricometodológicas qualitativas: a vivência da mulher no período reprodutivo. In: Pesquisa qualitativa em enfermagem, Rio de Janeiro (RJ): Guanabara Koogan; 2003. p. 1-3.

6. Organización Panamericana de la Salud. Investigación cualitativa en enfermería: contexto y bases conceptuales. Serie Paltex Salud y Sociedad. Washington D.C: OPAS; 2008, cap 8, p. 102.

7. Schütz A, Luckman T. Las estructuras del mundo de la vida. Buenos Aires (AR): Amorrortu; 1977.

8. Schütz A. El problema de la realidad social: escritos I. Buenos Aires (AR): Amorrortu; 1974a.

9. Schütz A. Estúdios sobre teoria social: escrito II. Buenos Aires (AR): Amorrortu; 1974b.

10. Schütz A. Fenomenologia del mundo social. Buenos Aires (AR): Paidós; 1972.

11. Coelho IM. Fenomenologia e educação. In: Coelho IM, Garnica AVM, Bicudo MAV, Cappeletti IF, organizadores. Fenomenologia: uma visão abrangente da educação. São Paulo (SP): Olho d'Água; 1999. p.53-104.

12. Merighi MAB, Hamano L, Cavalcante Lg. O exame preventivo do câncer cérvico-uterino: conhecimento 
e significado para as funcionárias de uma escola de enfermagem de uma instituição pública. Rev Esc Enferm USP. 2002 Set; 36(3):289-96.

13. Casate JC, Corrêa AK. Vivências de alunos de enfermagem em estágio hospitalar subsídios para refletir sobre a humanização em saúde. Rev Esc Enferm USP. 2006 Set; 40(3):321-8.

14. Meleis Al, Trangenstein PA. Facilitating transitions: redefinition of a nursing mission. Nurs Outlook. 1994 Nov-Dec; 42(6):255-9.

15. Newton JM, McKenna L. The transitional journey through the graduate year: a focus group study. Int J Nurs Stud. 2007 Sep [citada 2009 Sep 23]; 44(7):1231-7. Disponible en: http://www. journalofnursingstudies.com/article/S00207489(06)00170-2/abstract

16. Beecroft PC, Santner S, Lacy ML, Kunzman L, Dorey F. New graduate nurses' perceptions of mentoring: six-year programme evaluation. J Adv
Nurs. 2006 Sep [citada 2009 Sep 23]; 55(6):736-47. Disponible en: http:/ / www3.interscience.wiley. com/journal/118563537/abstract

17. Goran S.F. Mentorship as a teaching strategy. Crit Care Nurs Clin North Am. 2001 Mar; 13(1):119-29.

18. Anderson JK. The work-role transition of expert clinician to novice academic educator. J Nurs Educ. 2009 Apr; 48(4):203-8.

19. Cooper C, Taft L, Thelen AM. Preparing for practice: students \& apos; Reflections on their final clinical experience. J Prof Nurs. 2005 Sep-Oct; 21(5):293-302.

20. Santos M, Merighi MAB, Muñoz LA. La experiencia de las enfermeras clínicas con estudiantes de enfermería: un análisis fenomenológico. Rev Esc Enferm USP [online]. 2009 Sep [citada 2009 Sep 23]; 43(3):528-34. Disponible en: http:/ / www. scielo.br/scielo.php?script=sci_arttext\&pid=S0080$62342009000300005 \& \operatorname{lng}=\mathrm{en}$. 